Foundation Trust; ${ }^{9}$ Berkshire Healthcare NHS Foundation Trust; ${ }^{10}$ South London and Maudsley NHS Foundation Trust; ${ }^{11}$ 2gether NHS Foundation Trust; Justine Brennan-Tovey, Cumbria, Northumbria and Tees Valley NHS Foundation Trust; ${ }^{12}$ Oxleas NHS Foundation Trust; ${ }^{13}$ NHS Lothian; ${ }^{14}$ North East London NHS Foundation Trust; ${ }^{15}$ Greater Manchester Mental Health NHS Foundation Trust; ${ }^{16}$ Nottinghamshire Healthcare NHS Foundation Trust; ${ }^{17}$ Sheffield Health and Social Care NHS Foundation Trust; ${ }^{18}$ Avon and Wiltshire Mental Health Partnership NHS Trust; ${ }^{19}$ Hywel Dda University Health Board; ${ }^{20}$ Cheshire and Wirral Partnership NHS Foundation Trust;

${ }^{21}$ University of Leeds and ${ }^{22}$ Barnet, Enfield and Haringey Mental Health Trust and University College London

${ }^{*}$ Corresponding author.

doi: 10.1192/bjo.2021.823

Aims. The number of people over the age of 65 attending Emergency Departments (ED) in the United Kingdom (UK) is increasing. Those who attend with a mental health related problem may be referred to liaison psychiatry for assessment. Improving responsiveness and integration of liaison psychiatry in general hospital settings is a national priority. To do this psychiatry teams must be adequately resourced and organised. However, it is unknown how trends in the number of referrals of older people to liaison psychiatry teams by EDs are changing, making this difficult.

Method. We performed a national multi-centre retrospective service evaluation, analysing existing psychiatry referral data from EDs of people over 65. Sites were selected from a convenience sample of older peoples liaison psychiatry departments. Departments from all regions of the UK were invited to participate via the RCPsych liaison and older peoples faculty email distribution lists. From departments who returned data, we combined the date and described trends in the number and rate of referrals over a 7 year period.

Result. Referral data from up to 28 EDs across England and Scotland over a 7 year period were analysed ( $n=18828$ referrals). There is a general trend towards increasing numbers of older people referred to liaison psychiatry year on year. Rates rose year on year from 1.4 referrals per 1000 ED attenders ( $>65$ years) in 2011 to 4.5 in 2019 . There is inter and intra site variability in referral numbers per $1000 \mathrm{ED}$ attendances between different departments, ranging from $0.1-24.3$.

Conclusion. To plan an effective healthcare system we need to understand the population it serves, and have appropriate structures and processes within it. The overarching message of this study is clear; older peoples mental health emergencies presenting in ED are common and appear to be increasingly so. Without appropriate investment either in EDs or community mental health services, this is unlikely to improve.

The data also suggest very variable inter-departmental referral rates. It is not possible to establish why rates from one department to another are so different, or whether outcomes for the population they serve are better or worse. The data does however highlight the importance of asking further questions about why the departments are different, and what impact that has on the patients they serve.

The efficiency of the medical role within a Single Point of Access (SPA) Service in reducing the number of clinic appointments required

\author{
Anca Bradu* and Sam Nayrouz \\ West London NHS Trust \\ ${ }^{\star}$ Corresponding author.
}

doi: 10.1192/bjo.2021.824
Aims. The SPA service takes referrals from general practitioners (GPs), medical professionals, the London Ambulance service, the London Police, psychology and social services, and from patients themselves and their family members or support groups. Some of these referrals require input from secondary care, but others can be solved within primary care if given specialist advice, this minimizing the time spent by patients in the healthcare system and minimizing also the NHS costs.

Our aim was to evaluate the implementation of the Advice from Medics Service in a 1-year period.

Method. We examined a random sample of 200 referrals between 1st of April 2019 and 31st of March 2020 out of all referrals that were considered, after the triage, to be appropriate for an advice on treatment provided by the medics as an alternative to a clinic appointment in secondary care. We collected information from the electronic patient records regarding the dates of referrals, the senders of referrals, the type of referrals, the age and gender of patients and the reasons to be referred, and finally we analysed the outcome of the referrals and compared it with the action requested.

Result. Of the 200 referrals, 113 were for female patients and 87 for male patients. The age of patients was between 18 and 91 years old, with a median of 43 years old.

The person/authority making the referral was the GP in 179 cases, and others in 21 cases.

The referrers asked for review in 74 cases, urgent review in 2 cases, review and advice in 31 cases, only advice in 46 cases, and did not state the type of referral in 47 cases.

The primary pathology implied was affective in most of the cases (122), followed by psychotic (31) and neurotic (22), organic (8), of personality (5), hyperkinetic disorders (5), due to substance misuse (4), of psychological development (2) and learning disability (1).

The outcome of the referrals was as follows: 19 patients (9.5\%) were seen by the Crisis Team, $11(5.5 \%)$ were referred to other teams, 4 (2\%) did not engage with SPA, and the rest of 166 $(83 \%)$ referrals were solved with advice.

Conclusion. The outcome was extremely favourable as the majority of referrals requested medical review but most of them (83\%) were solved with specialist advice to GPs or other professionals, highlighting that the implementation of the Advice from Medics Service has been an improvement to the SPA.

\section{Implementing a physical healthcare clinic in a CAMHS neurodevelopmental population}

Helen Bruce ${ }^{1 *}$, Sharika Mansoor ${ }^{2}$ and Sacha Evans ${ }^{3}$

${ }^{1}$ UCL Division of Psychiatry; ${ }^{2}$ Barts Health NHS Trust \& East London Foundation Trust and ${ }^{3}$ Great Ormond Street Hospital ${ }^{\star}$ Corresponding author.

doi: 10.1192/bjo.2021.825

Aims. To establish a physical health clinic in a community CAMHS to monitor patients in the NDT who are on stimulant/ antipsychotic medication

To re-audit to assess adherence to physical health monitoring in accordance with guidelines

Background. Studies have indicated that people with severe mental illness have higher rates of mortality and are prone to development of physical health problems compared to the general population. Monitoring physical health is therefore important as it allows early detection and intervention where appropriate.

Method. 17 out of 120 patients in the NDT were identified as taking either an antipsychotic (8 patients) or stimulant medication 
(9 patients). Physical health data required were determined by local policy and the Maudsley guidelines.

Parents were invited to attend the clinic with their child through telephone calls. Height, weight, blood pressure and pulse were measured in the appointment. A blood test form was provided for parents to take to local outpatient phlebotomy services. A GP letter was sent with the results of the physical health check with a request to conduct an ECG and notify us of any abnormal results. Feedback forms were collected from parents to share their experience of attending the physical health clinic.

Five patients were identified as having difficulty attending the CAMHS clinic due to refusal/challenging behaviour. For three patients, school visits were organised to conduct a physical health check.

Result. The results from the second round of the audit indicate an overall improvement in the adherence to monitoring guidelines for antipsychotic and stimulant medication. This was particularly evident for the patients on antipsychotic medication. Feedback collected from parents regarding the service provided was also positive.

Conclusion. The physical health clinic identified challenges preventing 100\% compliance in all patients. This included difficulties with parents bringing their child to CAMHS due to challenging behaviour. In a few of the patients, it was possible to solve this issue by conducting a school visit.

It was also observed that there were multiple instances where challenging behaviour lead to inability to conduct certain tests including blood pressure, blood tests and ECG. Additional strategies should be considered to improve compliance.

A notable issue that also arose from the development of the physical health clinic was that it was unclear how to obtain an ECG at CAMHS.

Continuation of the clinic as well as extension to include patients within other teams at Tower Hamlets CAMHs would be recommended.

\section{Reducing admission time to Broadmoor High Secure Hospital - a case review \\ Maria Vittoria Capanna ${ }^{1 \star}$, Saima $\mathrm{Ali}^{1}$ and Robert Bates ${ }^{2}$ \\ ${ }^{1}$ West London NHS Trust and ${ }^{2}$ Broadmoor Hospital, Crowthorne Berks \\ ${ }^{\star}$ Corresponding author.}

doi: 10.1192/bjo.2021.826

Aims. Prolonged waiting times for admission to psychiatric hospital settings are a common and widespread issue. Delayed admissions may result in poorer outcomes due to prolonged mental suffering and delays in initiating treatment. Long waiting times also have a negative impact at a service level, impeding patient flow.

National guidance has been recently updated, recommending that patient transfers to secure services take no longer than 28 days from referral. These transfers are frequently affected by delays in admission, possibly resulting in increased risk to patients, staff and the public.

The aim of this project was to audit all referrals to Broadmoor High Secure Hospital in England within a one year period with special focus taken on calculating the time taken from referral to admission. We aimed to assess if there were any rate limiting steps which could be targeted to reduce time from referral to admission.

Method. We collected data and conducted a retrospective cohort review for all admissions from September 2019-September 2020.
Where available, information was obtained for each step of the referrals process. Individual patient records were reviewed where required.

Exclusion criteria: data withdrawn, transfers from other high secure services (HSS), incomplete data, "MOJ instruction" or urgent admission bypassing the process.

Result. 18 cases were excluded as per exclusion criteria. 46 patients were included in the study. 16 referrals originated from medium secure psychiatric hospitals, and 30 from prison.

The average time from referral to admission was 44.3 days. Admission of patients from MSUs was quicker, taking an average of 40.3 days when compared to prison referrals, which took 45.9 days.

The breakdown of timings for each step in the referrals process was calculated to determine if a rate limiting step could be identified.On average it took 2.1 working days to allocate a case to a clinician, 7.6 days for an assessment, 9.2 days to complete a report and 3.5 days to submit this to the admissions panel. The mean time from referral to the date of the panel hearing was 22.5 working days, and admission took a further 21.8 days on average.

Conclusion. The current average time to admission exceeds the new 28 day recommendation. This could both be due to the COVID-19 pandemic, and miscommunication about time targets. We will review the process and aim to reduce the time from referral to admission in line with new guidance.

\section{Elderly offenders at Wathwood Hospital: perspectives and practicalities}

Sidra Chaudhry* and Gwilym Hayes

Wathwood Hospital

${ }^{\star}$ Corresponding author.

doi: 10.1192/bjo.2021.827

Aims. The following project explores where Wathwood Hospital stands in provision of services to its elderly patients.

Background. The only dedicated forensic medium secure unit for elderly offenders in England is the St. Andrews medium secure unit in Northampton with only 17 beds. Due to the limited beds, other units must accommodate elderly patients, which raises the question whether these units can provide the appropriate services for this very vulnerable population.

Method. Inclusion Criteria:

Male

$>55$ years of age

Admitted from 2012 onwards (from when database was maintained)

Data were gathered using patient electronic records including index offence, mental disorder, physical health comorbidities and discharge destinations. Patient identifiable data were anonymized to protect their identities.

A staff survey was also conducted to find their perspective on managing elderly patients and whether Wathwood Hospital had the appropriate resources for elderly offenders in their area of work. Result. A total of 220 referrals were searched with only 9 patients $>55$ years. Index offenses, mental disorder diagnoses, physical comorbidities including cognitive assessments in the form of memory tests and brain imaging were also collated for identified patients from electronic patient records.

Index offences included violence against person, arson, homicide, robbery, threatening behaviour and dangerous driving and 